\title{
Anomalous dispersion of microcavity trion-polaritons
}

\author{
S. Dhara ${ }^{1,2 \dagger}$, C. Chakraborty ${ }^{3,4}$, K. M. Goodfellow ${ }^{1,4}$, L. Qiu ${ }^{1,4}$, T. A. O'Loughlin ${ }^{1}$, G. W. Wicks ${ }^{1,3}$, \\ Subhro Bhattacharjee ${ }^{5}$ and A. N. Vamivakas ${ }^{1,3,4,6 \star}$
}

The strong coupling of excitons to optical cavities has provided new insights into cavity quantum electrodynamics as well as opportunities to engineer nanoscale light-matter interactions ${ }^{1-6}$. Here we study the interaction between out-ofequilibrium cavity photons and both neutral and negatively charged excitons, by embedding a single layer of the atomically thin semiconductor molybdenum diselenide in a monolithic optical cavity based on distributed Bragg reflectors. The interactions lead to multiple cavity polariton resonances and anomalous band inversion for the lower, trion-derived, polariton branch-the central result of the present work. Our theoretical analysis reveals that many-body effects in an out-of-equilibrium setting result in an effective level attraction between the exciton-polariton and trion-polariton accounting for the experimentally observed inverted trion-polariton dispersion. Our results suggest a pathway for studying interesting regimes in quantum many-body physics yielding possible new phases of quantum matter m $^{711}$ as well as fresh possibilities for polaritonic device architectures ${ }^{12-15}$.

Coupled harmonic oscillators are ubiquitous in physics and an exemplary platform to observe them is with cavity excitonpolaritons ${ }^{1,2}$. Pioneering work reported planar cavity photon and quantum well exciton-polariton formation ${ }^{3,4}$. Since then a wide range of optical resonances have been coupled to excitonic resonances ${ }^{5,6}$. Studies have ranged from studying polariton amplification, condensation and interactions with a two-dimensional electron gas $^{7-11,16}$ to leveraging these quantum matter coherences for light generation ${ }^{17-19}$. But, in almost all the previous work, the focus has been on polaritons formed out of neutral excitons and photons, with little work on other exciton complexes ${ }^{20,21}$.

The past decade has witnessed the emergence of atomically thin transition metal dichalcogenides (TMDCs) that support strongly bound neutral ${ }^{22}$, charged exciton resonances ${ }^{23}$ and defect excitons ${ }^{24}$. TMDC materials exhibit large exciton binding energies, stabilizing them at room temperature $e^{25,26}$. Recent work demonstrated polariton formation $^{27}$, and shortly thereafter negatively charged excitons (trions) and polarons were coupled to cavity photons ${ }^{28,29}$. In this work we study cavity polariton physics with a device that supports dispersing and mutually interacting, out-of-equilibrium, cavity photons, neutral excitons and negatively charged excitons.

Figure 1a presents an illustration of our device architecture (see Methods) containing the single-layer TMDC, molybdenum diselenide $\left(\mathrm{MoSe}_{2}\right)$, at the cavity anti-node. Natural doping levels are such that the device supports both neutral excitons and trions that can couple with the cavity photon. A conventional approach to describe the above excitations in the system would involve modelling them in terms of bosonic oscillators, as shown in Fig. 1b. We would expect to observe three polariton branches, an upper, middle and lower polariton branch (UPB, MPB, LPB). Such theoretically expected dispersions are presented in Fig. 1c using known energy detunings from our experiments, and this was indeed observed in recent experiments ${ }^{21}$.

The previous are in contrast to our observation shown in Fig. 1d. Figure 1d is a measurement of our device's polariton dispersion. The dispersion of the lower trion-polariton branch is completely inverted (compare to Fig. 1c), which translates to a negative mass for the trion-polaritons in the neighbourhood of $k_{\|}=0$. This is the central observation of our work. In what follows we describe the physics resulting in this spectrum and find it depends on the fermionic nature of the trions and how they interact with cavity photons, the strong interaction between the trions, the MPB and electrons, and the out-of-equilibrium regime of the experiment. Note that the fundamental difference between the earlier observation ${ }^{30}$ of negative polariton mass and this work is that many-body interactions result in an anomalous trion-polariton dispersion, resulting in charged cavity polaritons exhibiting negative mass in the neighbourhood of $k_{\|}=0$.

In our system, the constituent resonances are the $\mathrm{MoSe}_{2}$ exciton, trion and the cavity photon. The bottom panel of Fig. 2a presents the measured photoluminescence (PL) spectrum of the single-layer $\mathrm{MoSe}_{2}$ flake on a $\mathrm{SiO}_{2}$ substrate. We observe a stress-induced redshift of $8 \mathrm{meV}$ for both the neutral and charged excitons when the monolayer is embedded inside the microcavity. The exciton (trion) linewidth is $7 \mathrm{meV}(9 \mathrm{meV})$. Reflectance spectroscopy of the unloaded cavity (top panel, Fig. 2a) identifies the spectral location of the cavity resonance and reveals a quality factor of 600 and a linewidth of $3 \mathrm{meV}$. A PL spectrum of the loaded cavity is presented in Fig. $2 b$.

To unmask the polariton dispersion an angle resolving measurement set-up was constructed that images the objective (numerical aperture, NA 0.7) Fourier plane onto a spectrometer (Fig. 3a). The raw angle-resolved spectrum is shown in Fig. 3b, where the colour represents the differentiated intensity of PL in $\log$ (the raw PL data are shown in the Supplementary Section 2). As shown in Fig. 3c, it is possible to measure PL spectra at specific values of in-plane momentum. Fits to each of the polariton resonances in the spectra of Fig. $3 \mathrm{c}$ map the full polariton dispersion relation as reported in Fig. 1d. We observe a large Rabi splitting

\footnotetext{
${ }^{1}$ The Institute of Optics, University of Rochester, Rochester, New York 14627, USA. ${ }^{2}$ Department of Physics, Indian Institute of Technology, Kharagpur 721302, India. ${ }^{3}$ Materials Science, University of Rochester, Rochester, New York 14627, USA. ${ }^{4}$ Center for Coherence \& Quantum Optics, University of Rochester, Rochester, New York 14627, USA. ${ }^{5}$ International Centre for Theoretical Sciences, Tata Institute of Fundamental Research, Bangalore 560089 , India. ${ }^{6}$ Department of Physics, University of Rochester, Rochester, New York 14627, USA. ${ }^{\dagger}$ These authors contributed equally to this work.

e-mail: nick.vamivakas@rochester.edu
} 
a

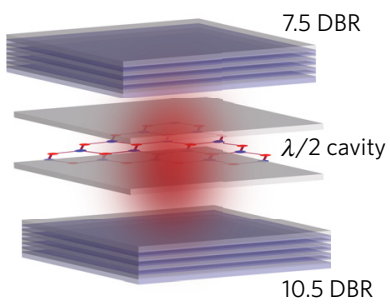

b

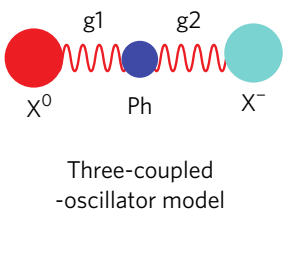

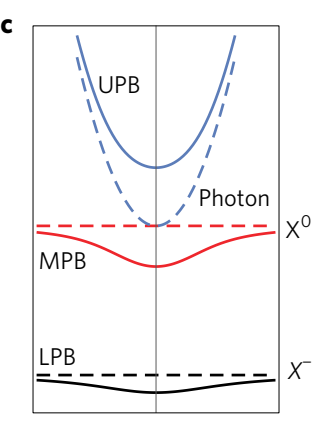

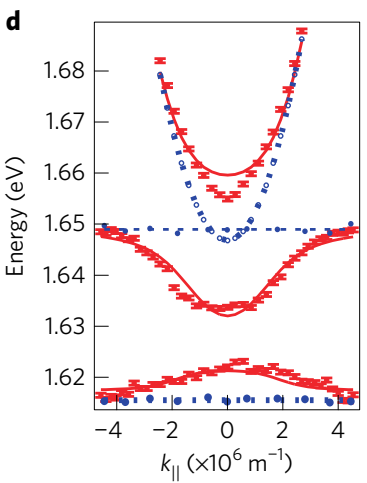

Figure 1 | Device architecture and cavity polariton physics. a, Schematic of the optical cavity. A monolayer of MoSe $e_{2}$ is embedded between the top and bottom distributed Bragg reflectors (DBRs). b, A coupled spring and mass system represents the three-coupled-oscillator problem involving the cavity photon (Ph), exciton and trion. g1 and g2 are the coupling constants. c, Dispersion relation for both the uncoupled cavity photon, exciton and trion (dashed lines) and for the coupled cavity photon, exciton and trion (solid lines) assuming three coupled oscillators as illustrated in $\mathbf{b}$. Notice the middle polariton branch (MPB) and lower polariton branch (LPB) exhibit positive dispersion. This is true irrespective of detuning between the cavity photon, exciton and trion. $\mathbf{d}$, A single-shot angle-resolved photoluminescence $(\mathrm{PL})$ measurement of our device exhibiting negative dispersion for the LPB. The solid line is the fit to our theoretical model. The flat dashed lines are the dispersion of the bare $\mathrm{MoSe}_{2}$ exciton and trion PL obtained at $6 \mathrm{~K}$ (no optical cavity) and the blue parabola is the bare cavity dispersion. Error bars denote resolution of the experimental set-up.

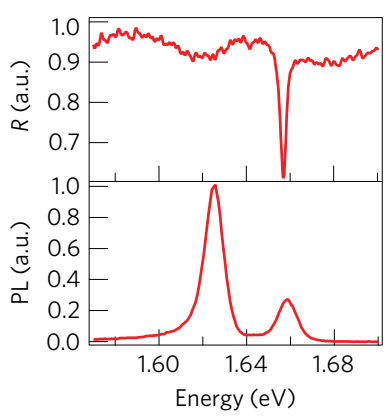

b

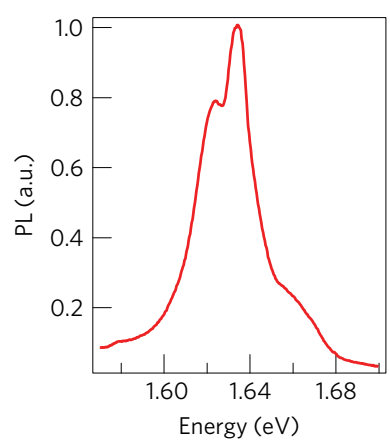

Figure 2 | Optical characterization of the cavity, $\mathrm{MoSe}_{2}$ flake and the polariton device. The same experimental set-up has been used to image the sample plane and to obtain the reflectance of the cavity at normal incidence without the $\mathrm{MoSe}_{2}$ flake using a low NA 0.13 objective. a, Top: Reflectance $(R)$ spectrum obtained at $5 \mathrm{~K}$ (NA 0.13). The cavity full-width at half-maximum (FWHM) is $\sim 3 \mathrm{meV}$. Bottom: PL from a bare $\mathrm{MoSe}_{2}$ sample at $5 \mathrm{~K}$ exhibiting both the neutral exciton and trion peaks (NA 0.7). b, The PL spectrum of the $\mathrm{MoSe}_{2}$ sample embedded in the cavity at $5 \mathrm{~K}$ shows the appearance of the upper $(1.66 \mathrm{eV})$, middle $(1.634 \mathrm{eV})$ and lower $(1.621 \mathrm{eV}$ ) polariton branches (NA 0.7).

of $\sim 25 \mathrm{meV}$ at zero in-plane momenta when the cavity photon and exciton are resonant (see Supplementary Section 1a).

To understand the microscopic mechanism responsible for the observed inverted dispersion relation and the negative trion mass, we introduce the following Hamiltonian written in momentum space

$$
\begin{aligned}
H= & \sum_{k} \Delta_{k}^{\mathrm{el}} c_{k}^{\dagger} c_{k}+\sum_{k, l} E_{k}^{l} \Gamma_{l k}^{\dagger} \Gamma_{l k}+\sum_{k} \omega_{k}^{\mathrm{tr}} \psi_{k}^{\dagger} \psi_{k} \\
& +v_{2} \sum_{k, q}\left(a_{k} c_{q} \psi_{k+q}^{\dagger}+\text { h.c. }\right)+v_{3} \sum_{k, q}\left(\Gamma_{2, k} c_{q} \psi_{k+q}^{\dagger}+\text { h.c. }\right)
\end{aligned}
$$

where $c_{k}^{\dagger}\left(c_{k}\right), a_{k}^{\dagger}\left(a_{k}\right), \psi_{k}^{\dagger}\left(\psi_{k}\right)$, and $\Gamma_{l, k}^{\dagger}\left(\Gamma_{l, k}\right)$ are the electron, photon, trion, upper $(l=1)$ and middle $(l=2)$ polariton creation (annihilation) operators, respectively; $\Delta_{k}^{\mathrm{el}}, \omega_{k}^{\mathrm{tr}}, E_{k}^{1}, E_{k}^{2}$, are the bare electron, trion, upper, and middle polariton resonance energies. $v_{2}$ quantifies the interaction strength of the cavity photon, electron and trion and $v_{3}$ quantifies the interaction strength of the middle polariton, the

electron and the trion (see Supplementary Fig. 1). In equation (1) we have neglected the interaction between the upper polariton and the trion as they are detuned from resonance. In this experiment, we have a small relative detuning between the cavity photon and the uncharged exciton and the trion is detuned by $30 \mathrm{meV}$ below the cavity resonance.

Assuming that the free electron concentration is such that the majority of free electrons form trions with the optically excited excitons $^{31}$, we integrate out the electronic contribution in the model. To the leading order in perturbation theory in $v_{2}$ and $v_{3}$ (see Supplementary Section $1 \mathrm{~b}$ ), this generates interaction between the photons and the trions, between the middle polariton branch and the trions, as well as mutual interaction among the photons, middle polariton branch and the trions. In addition, it also renormalizes the bare dispersion of the trion. Now, to account only for the dispersion, we make a mean-field approximation by introducing mean-field occupation numbers for the trions and the polaritons: $\left\langle\psi_{k^{\prime}}^{\dagger} \psi_{k}\right\rangle=\delta_{k^{\prime} k} n_{t k}$ and $\left\langle\Gamma_{a k}^{\dagger} \Gamma_{b k^{\prime}}\right\rangle=\delta_{k k^{\prime}} \delta_{a b} n_{\Gamma_{a, k}}$, where $n_{t k}$ and $n_{\Gamma_{a, k}}$ are the respective out-of-equilibrium occupation numbers for the trion and upper $(a=1)$ and middle $(a=2)$ polariton modes. Since such out-of-equilibrium occupancy is expected to be very different from the equilibrium Bose-Einstein (for polaritons) or Fermi-Dirac (for trions) distributions, within mean-field theory we take the ratio of occupancies of the MPB and UPB as a $k$-independent fitting parameter. The data in Fig. $1 \mathrm{~d}$ are fitted by a ratio of $\sim 1.3$ in accordance with the PL data (see Fig. 2b).

The mean-field Hamiltonian (see Supplementary Section 1d) can be diagonalized to yield the dispersion relation. The data in Fig. 1d are fitted by our model (seen Supplementary Table 1). In particular, for the trion-polariton branch our mean-field calculations yield the following dispersion

$$
E_{\mathrm{tr}}(k)=E_{\mathrm{tr}}^{0}-E\left(\alpha_{k}, \beta_{k}\right)
$$

where $\alpha_{k}$ and $\beta_{k}$ (see Supplementary Section 1c) are two matrix elements that transform the exciton-photon basis to the polariton basis, $E_{\mathrm{tr}}^{0}$ is the bare trion energy and $E\left(\alpha_{k}, \beta_{k}\right)$ captures the many-body effects present in our device. The sign of $E\left(\alpha_{k}, \beta_{k}\right)$ is determined by the relative sign of the coupling constants $v_{2}$ and $v_{3}$, and we find the relative sign of $v_{2}$ and $v_{3}$ has to be opposite to give the hybridization.

We can understand the above inverted trion-polariton dispersion as resulting from an electron-mediated interaction between the 

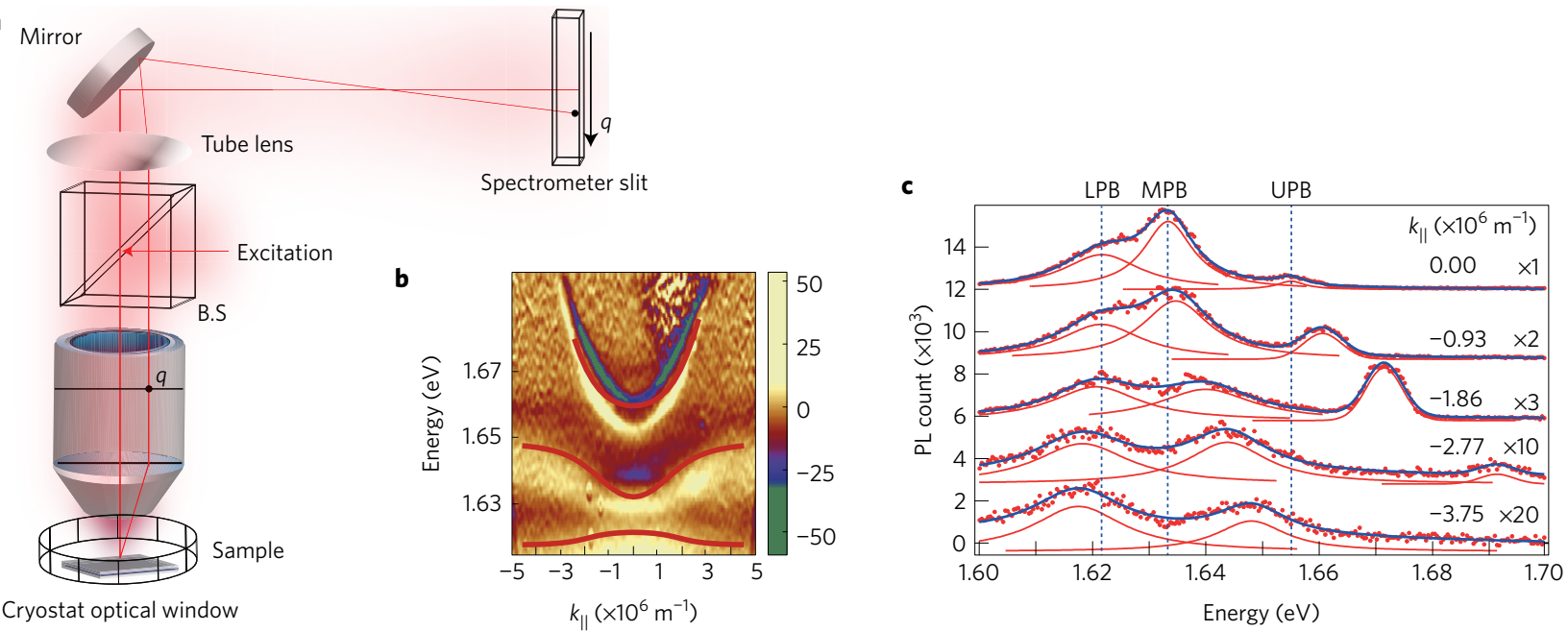

Figure 3 | Measuring the device dispersion relation. a, Schematic of the optical microscope set-up for the angle-resolved measurements. A beam splitter is used to excite the sample through a high NA (0.7) objective lens and a tube lens is placed to image the Fourier plane of the objective directly on to the imaging spectrometer. $\mathbf{b}, \mathrm{A}$ colour scale plot of the angle-resolved PL spectrum obtained at $6 \mathrm{~K}$; the logarithm of the raw data is differentiated to enhance the contrast. The solid lines are the fitting obtained from our model as discussed in the text. c, PL spectrum as taken from the line cuts at five different $k_{\|}$ obtained from the raw data which are presented in Supplementary Section 2.
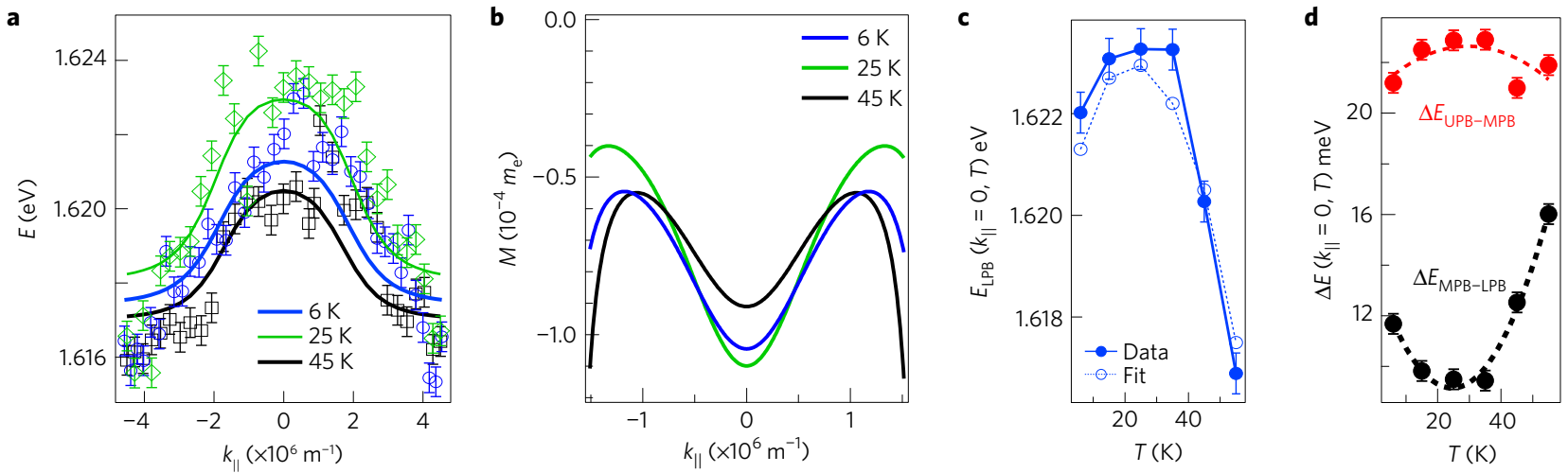

Figure 4 | Temperature dependence of the interactions. a, Lower polariton branch dispersion at three temperature points in the range for $6 \mathrm{~K}$ to $45 \mathrm{~K}$. The curvature and position of each trion-polariton branch is a function of the detuning between the exciton resonance and cavity photon, which changes with the temperature as discussed in the text. $\mathbf{b}$, LPB effective mass near zero in-plane momentum values for the same temperature points as in $\mathbf{a}$. $\mathbf{c}$, Plot of the trion-polariton resonance at $k_{\|}=0$ as a function of temperature. Solid (open) circles represent data points obtained from the raw data (fitting of the model). Note that the resonance energy is blue shifted and reaches its maximum near $25-30 \mathrm{~K}$ when the exciton-cavity photon detuning is minimum. d, Plot of the energy difference between the upper polariton and middle polariton branch at $k_{\|}=0$ ( $\left.\Delta E_{U P B}-M P B\right)$ (red) with maximum level repulsion near $25-30 \mathrm{~K}$, leading to maximum level attraction for the energy difference between the middle polariton and lower polariton branch at $k_{\|}=0$ ( $\Delta E_{\mathrm{MPB}-\mathrm{LPB}}$ ) (black) near the same temperature. Dashed lines are guides to the eye. Error bars denote resolution of the experimental set-up.

exciton-polariton branch and the trion-polariton that overwhelms the weak trion cavity repulsion, leading to level attraction. The origin of such an interaction may be understood in terms of exchange of an electron between a neutral exciton and the negatively charged trion, and thus gaining delocalization energy of the trion's electron. Note in ref. 32 that the combination of increased sample doping and zero cavity-trion detuning overwhelms the many-bodyinteraction-induced inverted dispersion observed in Fig. 1d.

To demonstrate the level attraction is controllable we vary the device temperature. Figure $4 \mathrm{a}$ shows data for the temperaturedependent trion-polariton dispersion branch $(T=6 \mathrm{~K}, 25 \mathrm{~K}$ and $45 \mathrm{~K})$. In this temperature range the bare exciton is first red-detuned from the cavity $(T=6 \mathrm{~K})$, brought into resonance near $25 \mathrm{~K}$, and then becomes blue-detuned from the cavity for higher temperatures (above $55 \mathrm{~K}$ the trion becomes unstable). The tunability of the LPB negative mass, determined via $m_{\text {eff }}^{-1}=\hbar^{-2}\left(\mathrm{~d}^{2} E / \mathrm{d} k^{2}\right)$, in the vicinity of low $k_{\|}$for different temperatures is presented in Fig. $4 \mathrm{~b}$ (see Supplementary Section 3). In Fig. 4c, the trion-polariton zero momentum energy $E_{\mathrm{tr}}(0)$ is plotted. The solid (open) circles present the raw data (model). As the temperature is increased, the exciton is brought into resonance with the optical cavity and the level hybridization between the exciton-polariton and the trion-polariton is enhanced. This is again seen in Fig. $4 \mathrm{~d}$, where the energy difference at zero inplane momentum between the UPB and $\mathrm{MPB}\left(\Delta E_{\mathrm{UPB}-\mathrm{MPB}}\right)$ and $\mathrm{MPB}$ and LPB $\left(\Delta E_{\mathrm{MPB}-\mathrm{LPB}}\right)$ is plotted as a function of temperature.

The ease with which atomically thin materials can be doped and incorporated in photonic devices will provide many opportunities to study Fermi polaritons in the solid state. Future work will involve controlled in situ doping and resonant, angle-sensitive polariton pumping. Further, the combination of the magnitude and sign of the lower polariton branch effective mass and its extra charge provide a unique opportunity to build responsive current-carrying polaritonic 
circuitry that exhibits anomalous dissipative behaviour. We intend to explore how the negative mass influences the effect of disorder on the flow of the observed negative mass charged polaritons.

\section{Methods}

Methods, including statements of data availability and any associated accession codes and references, are available in the online version of this paper.

Received 19 April 2017; accepted 27 September 2017; published online 30 October 2017

\section{References}

1. Kavokin, A. V., Baumberg, J. J., Malpuech, G. \& Laussy, F. P. Microcavities (Oxford Science Publication, 2007).

2. Gibbs, H. M., Khitrova, G. \& Koch, S. W. Exciton-polariton light-semiconductor coupling effects. Nat. Photon. 5, 273 (2011).

3. Weisbuch, C., Nishioka, M., Ishikawa, A. \& Arakawa, Y. Observation of the coupled exciton-photon mode splitting in a semiconductor quantum microcavity. Phys. Rev. Lett. 69, 3314-3317 (1992).

4. Pau, S., Björk, G., Jacobson, J., Cao, H. \& Yamamoto, Y. Microcavity exciton-polariton splitting in the linear regime. Phys. Rev. B 51, 14437-14447 (1995).

5. Lidzey, D. G. et al. Strong exciton-photon coupling in an organic semiconductor microcavity. Nature 395, 53-55 (1998).

6. Hennessy, K. et al. Quantum nature of a strongly coupled single quantum dot-cavity system. Nature 445, 896-899 (2007).

7. Balili, R., Hartwell, V., Snoke, D., Pfeiffer, L. \& West, K. Bose-Einstein condensation of microcavity polaritons in a trap. Science $\mathbf{3 1 6}$, 1007-1010 (2007).

8. Deng, H., Haug, H. \& Yamamoto, Y. Exciton-polariton Bose-Einstein condensation. Rev. Mod. Phys. 82, 1489-1537 (2010).

9. Plumhof, J. D., Stöferle, T., Mai, L., Scherf, U. \& Mahrt, R. F. Room-temperature Bose-Einstein condensation of cavity exciton-polaritons in a polymer. Nat. Mater. 13, 247-252 (2013).

10. Byrnes, T., Kim, N. Y. \& Yamamoto, Y. Exciton-polariton condensates. Nat. Phys. 10, 803-813 (2014).

11. Smolka, S. et al. Cavity quantum electrodynamics with many-body states of a two-dimensional electron gas. Science 346, 332-335 (2014).

12. Deng, H., Weihs, G., Snoke, D., Bloch, J. \& Yamamoto, Y. Polariton lasing vs. photon lasing in a semiconductor microcavity. Proc. Natl Acad. Sci. USA 100, 15318-15323 (2003).

13. Tsintzos, S. I., Pelekanos, N. T., Konstantinidis, G., Hatzopoulos, Z. \& Savvidis, P. G. A GaAs polariton light-emitting diode operating near room temperature. Nature 453, 372-375 (2008)

14. Ballarini, D. et al. All-optical polariton transistor. Nat. Commun. 4, 1778 (2013).

15. Cerna, R. et al. Ultrafast tristable spin memory of a coherent polariton gas. Nat. Commun. 4, 2008 (2013).

16. Savvidis, P. G. et al. Angle-resonant stimulated polariton amplifier. Phys. Rev. Lett. 84, 1547-1550 (2000).

17. Kéna-Cohen, S. \& Forrest, S. R. Room-temperature polariton lasing in an organic single-crystal microcavity. Nat. Photon. 4, 371-375 (2010).

18. Das, A. et al. Room temperature ultralow threshold GaN nanowire polariton laser. Phys. Rev. Lett. 107, 066405 (2011).
19. Schneider, C. et al. An electrically pumped polariton laser. Nature 497, 348-352 (2013)

20. Rapaport, R., Cohen, E., Ron, A., Linder, E. \& Pfeiffer, L. N. Negatively charged polaritons in a semiconductor microcavity. Phys. Rev. B 63, 235310 (2001).

21. Flatten, L. C. et al. Strong exciton-photon coupling with colloidal nanoplatelets in an open microcavity. Nano Lett. 16, 7137-7141 (2016).

22. Wang, Q. H., Kalantar-Zadeh, K., Kis, A., Coleman, J. N. \& Strano, M. S. Electronics and optoelectronics of two-dimensional transition metal dichalcogenides. Nat. Nanotech. 7, 699-712 (2012).

23. Mak, K. F. et al. Tightly bound trions in monolayer $\mathrm{MoS}_{2}$. Nat. Mater. 12, 207-211 (2012)

24. Chakraborty, C., Kinnischtzke, L., Goodfellow, K. M., Beams, R. \& Vamivakas, A. N. Voltage-controlled quantum light from an atomically thin semiconductor. Nat. Nanotech. 10, 507-511 (2015).

25. Ramasubramaniam, A. Large excitonic effects in monolayers of molybdenum and tungsten dichalcogenides. Phys. Rev. B 86, 115409 (2012).

26. Ugeda, M. M. et al. Giant bandgap renormalization and excitonic effects in a monolayer transition metal dichalcogenide semiconductor. Nat. Mater. 13, 1091-1095 (2014)

27. Liu, X. et al. Strong light-matter coupling in two-dimensional atomic crystals. Nat. Photon. 9, 30-34 (2014).

28. Dufferwiel, S. et al. Exciton-polaritons in van der Waals heterostructures embedded in tunable microcavities. Nat. Commun. 6, 8579 (2015).

29. Sidler, M. et al. Fermi polaron-polaritons in charge-tunable atomically thin semiconductors. Nat. Phys. 13, 255-261 (2016).

30. Sich, M. et al. Observation of bright polariton solitons in a semiconductor microcavity. Nat. Photon. 6, 50-55 (2011).

31. Ross, J. S. et al. Electrical control of neutral and charged excitons in a monolayer semiconductor. Nat. Commun. 4, 1474 (2013).

32. Lundt, N. et al. Valley polarized relaxation and upconversion luminescence from Tamm-plasmon trion-polaritons with a $\mathrm{MoSe}_{2}$ monolayer. $2 D$ Mater. 4, 025096 (2017)

\section{Acknowledgements}

This work was supported by NSF EFRI EFMA-1542707, NSF CAREER DMR 1553788 , AFOSR FA9550-16-1-0020 and the University of Rochester University Research Award and the Leonard Mandel Faculty Fellowship in Quantum Optics. S.D. also acknowledges support from a Ramanujan Fellowship research grant, SERB, and ISIRD project SRIC, IIT Kharagpur. S.B. acknowledges the Max-Planck-Gesellschaft for funding though MPI partner group at ICTS.

\section{Author contributions}

S.D., C.C., K.M.G., T.A.O'L., G.W.W. and A.N.V. conceived the research. S.D., C.C., T.A.O'L. and K.M.G. fabricated the samples. S.D., C.C. and L.Q. conducted the measurements. S.D., C.C., S.B. and A.N.V. devised the theoretical model. All authors discussed the data and wrote the manuscript.

\section{Additional information}

Supplementary information is available in the online version of the paper. Reprints and permissions information is available online at www.nature.com/reprints. Publisher's note: Springer Nature remains neutral with regard to jurisdictional claims in published maps and institutional affiliations. Correspondence and requests for materials should be addressed to A.N.V.

\section{Competing financial interests}

The authors declare no competing financial interests. 


\section{Methods}

We deposit silicon dioxide $\left(\mathrm{SiO}_{2}\right)$ and tantalum pentoxide $\left(\mathrm{Ta}_{2} \mathrm{O}_{5}\right)$ as two dielectrics with refractive indices of 1.45 and 2.10, respectively, by electron beam evaporation to fabricate the distributed Bragg reflector (DBR). The thickness of each layer of oxide film (controlled by the thickness monitor) is $\lambda / 4 n$, where $\lambda$ and $n$ are the resonance wavelength of the cavity and the refractive index of the dielectric. First, a $\lambda / 4 n$ thick (half of the total cavity thickness) $\mathrm{SiO}_{2}$ film is deposited on top of the bottom mirror, then we deposit a mechanically exfoliated monolayer of $\mathrm{MoSe}_{2}$ layer by a dry transfer technique. Subsequently, we deposit the other half of the cavity and the rest of the top mirror. A $675 \mathrm{~nm}(1.836 \mathrm{eV})$ continuous wave laser was used to excite the sample from the top mirror in a microscopy cryostat. An empty cavity was used to calibrate the charge-coupled device (CCD) pixel to sample momentum in-plane via the relation $k_{\|}=k_{0} \sin (\theta)$. The reflectivities of the top and bottom mirrors were designed to have a slight asymmetry by choosing a different number of pairs for the top (7.5) and bottom (10.5) mirrors.

Data availability. The data that support the plots within this paper and other findings of this study are available from the corresponding author upon reasonable request. 